\title{
EXAMINATION OF THE WEED FLORA OF RUDER HABITATS AND THE POSSIBILITY OF SUPPRESSION WITH HERBICIDES
}

\author{
Milić Vojinović ${ }^{1}$, Jelica Živić ${ }^{1}$, Sanja Perić ${ }^{1}$, Miroljub Aksić ${ }^{2}$ \\ ${ }^{1}$ College of Agriculture and Food Technology, Ćirila i Metodija 1, 18400 Prokuplje, Serbia \\ phone: +38127/324-311, +381698702553, e-mail: vojinovic63@gmail.com \\ ${ }^{2}$ Faculty of Agriculture, Kosovska Mitrovica
}

\begin{abstract}
Ruderal flora, as well as the vegetation that flora forms, represent an extremely dynamic floristic-vegetation complex and arean integral part of the most immediate living and working environment of human. It is formed and developed mainly in human settlements, as well as in the other anthropogenic environments that are occasionally or permanently under direct or indirect influence of various forms of human activity. Ruderal vegetation is found not only directly around the settlements, but also around all urban and accompanying facilities: along roads, paths and fences around houses, yards, walls and roofs, in avenues, on ruins, construction sites, landfills, along railway tracks, road and defense embankments, on wet and nitrified banks of rivers, near human settlements, in abandoned lawns, on the street walks with sandy areas, cemeteries, in degraded pastures, forests, etc. This essay presents the distribution and representation of economically harmful, invasive and quarantine weed species (Abutilon theophrasti, Agropyrumrepens, Amaranthusretroflexus, Calystegiasepium, Cirsiumarvense, Chenopodium album, Chenopodiumhybridum, Convolvulus arvensis, Cynodondactylon, Daturastramonium, Sonchusarvensis, Sorghum halepense, Xanthium strumarium...) at ten sites in the Nisava district. The assessment of species representation was done in two shootings (May and August) according to scale 1-4. The proper selection of herbicides depends, in a large extent, on the presence of dominant weed species and on the time of application.

Keywords: weed flora, ruderal habitats, distribution, herbicides.
\end{abstract}

\section{INTRODUCTION}

Ruderal flora, as well as the vegetation that flora forms, represent an extremely dynamic floristic-vegetation complex and arean integral part of the most immediate living and working environment of human. It is formed and developed mainly in human settlements, as well as in the other anthropogenic environments that are occasionally or permanently under direct or indirect influence of various forms of human activity. Ruderal vegetation is found not only directly around the settlements, but also around all urban and accompanying facilities: along roads, paths and fences around houses, yards, walls and roofs, in avenues, on ruins, construction sites, landfills, along railway tracks, road and defense embankments, on wet and nitrified banks of rivers, near human settlements, in abandoned lawns, on the street walks with sandy areas, cemeteries, in degraded pastures, forests, etc.

\section{MATERIAL AND METHODES}

The experiment was set up in ten locations in the Nisava district. The distribution and representation of economically harmful, invasive and quarantine Weed species (Abutilon theophrasti, Agropyrumrepens, Amaranthusretroflexus, Calystegiasepium, Cirsiumarvense, Chenopodium album, Chenopodiumhybridum, Convolvulus arvensis, Cynodondactylon, Daturastramonium, Sonchusarvensis, Sorghum halepense, Xanthium strumarium)have been observed. The assessment of species representation was done in two shootings (May and August) according to scale 1-4.

\section{IRTIIE Vol. 6, No. 4, 2018 ISSN 1314-8788 (print), ISSN 1314-8796 (online), doi: 10.15547/artte.2018.04.007}




\section{RESULTS OF WORK}

At all ten sites, the presence of the following weeds was noticed: Abutilon theophrasti, Agropyrumrepens, Amaranthusretroflexus, Calystegiasepium, Cirsiumarvense, Chenopodium album, Chenopodiumhybridum, Convolvulus arvensis, Cynodondactylon, Daturastramonium, Sonchusarvensis, Sorghum halepense, Xanthium strumarium, and the assessment of representation according to scale 1-4 is given in Table 1.

Table 1.Representation of weeds according to scale 1-4 and according to phases of weeds growth

\begin{tabular}{|l|c|c|}
\hline \multicolumn{1}{|c|}{ Weed } & $\begin{array}{c}\text { Evaluation of } \\
\text { representation }\end{array}$ & $\begin{array}{c}\text { Phase of weed } \\
\text { development } \\
\text { s-small plant } \\
\text { a-adult plant }\end{array}$ \\
\hline Abutilon theophrasti & 2 & $\mathrm{~s}-\mathrm{a}$ \\
\hline Agropyrumrepens & 3 & $\mathrm{~s}-\mathrm{a}$ \\
\hline Amaranthusretroflexus & 3 & $\mathrm{~s}-\mathrm{a}$ \\
\hline Calystegiasepium & 1 & $\mathrm{~s}-\mathrm{a}$ \\
\hline Cirsium arvense & 4 & $\mathrm{~s}-\mathrm{a}$ \\
\hline Chenopodium album & 3 & $\mathrm{~s}-\mathrm{a}$ \\
\hline Chenopodium hybridum & 1 & $\mathrm{~s}-\mathrm{a}$ \\
\hline Convolvulus arvensis & 2 & $\mathrm{~s}-\mathrm{a}$ \\
\hline Cynodon dactylon & 3 & $\mathrm{~s}-\mathrm{a}$ \\
\hline Datura stramonium & 2 & $\mathrm{~s}-\mathrm{a}$ \\
\hline Sonchus arvensis & 2 & $\mathrm{~s}-\mathrm{a}$ \\
\hline Sorghum halepense & 2 & $\mathrm{~s}-\mathrm{a}$ \\
\hline Xanthium strumarium & 4 & $\mathrm{~s}-\mathrm{a}$ \\
\hline
\end{tabular}

The appropriate choice of herbicides, mostly depends on the presence of dominant weed species as well as of the time of their use. Herbicides of weed suppression in ruderal habitats are shown in Table 2.

Table 2. Herbicides of weed suppression in ruderal habitats

\begin{tabular}{|c|c|c|}
\hline \multirow[t]{2}{*}{ Weed } & \multicolumn{2}{|c|}{ Herbicides } \\
\hline & Destroyes weed great & Destroyes weed well \\
\hline Abutilon theophrasti & $\begin{array}{l}\text { glifosat (Glifosat, Glifosat } \\
\text { SL-480, Glifomark, Pirokor, } \\
\text { Roundup, Bingo, Cosmic, } \\
\text { Apsolut, Glifohem), } \\
\text { glufosinatamonijum (Basta- } \\
\text { 15, Rugby, Cunami), dikvat } \\
\text { (Dessicach,Diqua, Galop) }\end{array}$ & $\begin{array}{l}\text { 2,4-D } \quad \text { (Maton, } \\
\text { Velox, } \\
\text { Lentemul) }\end{array}$ \\
\hline Agropyrumrepens & $\begin{array}{l}\text { glifosat (Glifosat, Glifosat } \\
\text { SL-480, Glifomark, Pirokor, } \\
\text { Roundup, Bingo, Cosmic, } \\
\text { Apsolut, Glifohem), } \\
\text { glufosinatamonijum (Basta- } \\
\text { 15, Rugby, Cunami), dikvat } \\
\text { (Dessicach,Diqua, Galop) }\end{array}$ & $\begin{array}{lrr}\text { 2,4-D } & \text { (Maton, } & \text { Monosanherbi, } \\
\text { Velox, } & \text { Mustang, } & \text { Esteron, } \\
\text { Lentemul) } & & \end{array}$ \\
\hline
\end{tabular}




\begin{tabular}{|c|c|c|}
\hline Amaranthusretroflexus & $\begin{array}{l}\text { glifosat (Glifosat, Glifosat } \\
\text { SL-480, Glifomark, Pirokor, } \\
\text { Roundup, Bingo, Cosmic, } \\
\text { Apsolut, Glifohem), } \\
\text { glufosinatamonijum (Basta- } \\
\text { 15, Rugby, Cunami), dikvat } \\
\text { (Dessicach,Diqua, Galop) }\end{array}$ & $\begin{array}{lrr}\text { 2,4-D } & \text { (Maton, } & \text { Monosanherbi, } \\
\text { Velox, } & \text { Mustang, } & \text { Esteron, } \\
\text { Lentemul) } & & \end{array}$ \\
\hline Calystegiasepium & $\begin{array}{l}\text { glifosat (Glifosat, Glifosat } \\
\text { SL-480, Glifomark, Pirokor, } \\
\text { Roundup, Bingo, Cosmic, } \\
\text { Apsolut, Glifohem), } \\
\text { glufosinatamonijum (Basta- } \\
\text { 15, Rugby, Cunami), dikvat } \\
\text { (Dessicach,Diqua, Galop) }\end{array}$ & $\begin{array}{l}\text { 2,4-D (Maton, Monosanherbi, } \\
\text { Velox, Mustang, Esteron, } \\
\text { Lentemul) } \\
\text { Also herbicides used at crops can } \\
\text { be used, but in bigger quantity }\end{array}$ \\
\hline Cirsium arvense & $\begin{array}{l}\text { glifosat (Glifosat, Glifosat } \\
\text { SL-480, Glifomark, Pirokor, } \\
\text { Roundup, Bingo, Cosmic, } \\
\text { Apsolut, Glifohem), } \\
\text { glufosinatamonijum (Basta- } \\
\text { 15, Rugby, Cunami), dikvat } \\
\text { (Dessicach,Diqua, Galop) }\end{array}$ & $\begin{array}{l}\text { 2,4-D (Maton, Monosanherbi, } \\
\begin{array}{l}\text { Velox, Mustang, Esteron, } \\
\text { Lentemul) }\end{array} \\
\text { Also herbicides used at crops can } \\
\text { be used, but in bigger quantity }\end{array}$ \\
\hline $\begin{array}{l}\text { Chenopodium album } \\
\text { Chenopodium } \\
\text { hybridum }\end{array}$ & $\begin{array}{l}\text { glifosat (Glifosat, Glifosat } \\
\text { SL-480, Glifomark, Pirokor, } \\
\text { Roundup, Bingo, Cosmic, } \\
\text { Apsolut, Glifohem), } \\
\text { glufosinatamonijum (Basta- } \\
\text { 15, Rugby, Cunami), dikvat } \\
\text { (Dessicach,Diqua, Galop) }\end{array}$ & $\begin{array}{l}\text { 2,4-D (Maton, Monosanherbi, } \\
\begin{array}{l}\text { Velox, Mustang, Esteron, } \\
\text { Lentemul) }\end{array} \\
\text { Also herbicides used at crops can } \\
\text { be used, but in bigger quantity }\end{array}$ \\
\hline Convolvulus a & $\begin{array}{l}\text { glifosat (Glifosat, Glifosat } \\
\text { SL-480, Glifomark, Pirokor, } \\
\text { Roundup, Bingo, Cosmic, } \\
\text { Apsolut, Glifohem), } \\
\text { glufosinatamonijum (Basta- } \\
\text { 15, Rugby, Cunami), dikvat } \\
\text { (Dessicach,Diqua, Galop) }\end{array}$ & $\begin{array}{l}\text { 2,4-D (Maton, Monosanherbi, } \\
\begin{array}{l}\text { Velox, Mustang, Esteron, } \\
\text { Lentemul) }\end{array} \\
\text { Also herbicides used at crops can } \\
\text { be used, but in bigger quantity }\end{array}$ \\
\hline Cyn & $\begin{array}{l}\text { glifosat (Glifosat, Glifosat } \\
\text { SL-480, Glifomark, Pirokor, } \\
\text { Roundup, Bingo, Cosmic, } \\
\text { Apsolut, Glifohem), } \\
\text { glufosinatamonijum (Basta- } \\
\text { 15, Rugby, Cunami), dikvat } \\
\text { (Dessicach,Diqua, Galop) }\end{array}$ & $\begin{array}{l}\text { 2,4-D (Maton, Monosanherbi, } \\
\text { Velox, Mustang, Esteron, } \\
\text { Lentemul) } \\
\text { Also herbicides used at crops can } \\
\text { be used, but in bigger quantity }\end{array}$ \\
\hline Datura stramonium & $\begin{array}{l}\text { glifosat (Glifosat, Glifosat } \\
\text { SL-480, Glifomark, Pirokor, } \\
\text { Roundup, Bingo, Cosmic, } \\
\text { Apsolut, Glifohem), } \\
\text { glufosinatamonijum (Basta- } \\
\text { 15, Rugby, Cunami), dikvat } \\
\text { (Dessicach,Diqua, Galop) }\end{array}$ & $\begin{array}{l}\text { 2,4-D (Maton, Monosanherbi, } \\
\begin{array}{l}\text { Velox, Mustang, Esteron, } \\
\text { Lentemul) }\end{array} \\
\text { Also herbicides used at crops can } \\
\text { be used, but in bigger quantity }\end{array}$ \\
\hline
\end{tabular}




\begin{tabular}{|c|c|c|}
\hline Sonchus arvensis & $\begin{array}{l}\text { glifosat (Glifosat, Glifosat } \\
\text { SL-480, Glifomark, Pirokor, } \\
\text { Roundup, Bingo, Cosmic, } \\
\text { Apsolut, Glifohem), } \\
\text { glufosinatamonijum (Basta- } \\
\text { 15, Rugby, Cunami), dikvat } \\
\text { (Dessicach,Diqua, Galop) }\end{array}$ & $\begin{array}{l}\text { 2,4-D (Maton, Monosanherbi, } \\
\text { Velox, Mustang, Esteron, } \\
\text { Lentemul) }\end{array}$ \\
\hline Sorghum halepense & $\begin{array}{l}\text { glifosat (Glifosat, Glifosat } \\
\text { SL-480, Glifomark, Pirokor, } \\
\text { Roundup, Bingo, Cosmic, } \\
\text { Apsolut, Glifohem), } \\
\text { glufosinatamonijum (Basta- } \\
\text { 15, Rugby, Cunami), dikvat } \\
\text { (Dessicach,Diqua, Galop) }\end{array}$ & $\begin{array}{l}\text { 2,4-D (Maton, Monosanherbi, } \\
\text { Velox, Mustang, Esteron, } \\
\text { Lentemul) } \\
\text { Also herbicides used at crops can } \\
\text { be used, but in bigger quantity }\end{array}$ \\
\hline Xanthium strumarium & $\begin{array}{l}\text { glifosat (Glifosat, Glifosat } \\
\text { SL-480, Glifomark, Pirokor, } \\
\text { Roundup, Bingo, Cosmic, } \\
\text { Apsolut, Glifohem), } \\
\text { glufosinatamonijum (Basta- } \\
\text { 15, Rugby, Cunami), dikvat } \\
\text { (Dessicach,Diqua, Galop) }\end{array}$ & $\begin{array}{l}\text { 2,4-D (Maton, Monosanherbi, } \\
\text { Velox, Mustang, Esteron, } \\
\text { Lentemul) } \\
\text { Also herbicides used at crops can } \\
\text { be used, but in bigger quantity }\end{array}$ \\
\hline
\end{tabular}

\section{CONCLUSION}

According to obtained and shown results, the most frequent weeds on ruderal habitats at ten sites in Nisava district are Abutilon theophrasti, Agropyrumrepens, Amaranthusretroflexus, Calystegiasepium, Cirsiumarvense, Chenopodium album, Chenopodiumhybridum, Convolvulus arvensis, Cynodondactylon, Daturastramonium, Sonchusarvensis , Sorghum halepense, Xanthium strumarium with a score of 1-4. Present weeds on ruderal habitats are in the phase of the pony and in the phase of adult plants.

Chemical agents that suppress above mentioned weeds are based on active substance Glyphosate with Glifosat, Glifosat SL-480, Glifomark, Pirokor, Roundup, Bingo, Cosmic, Apsolut, Glifohem preparations, and active substance glufosinate ammonium with Basta-15, Rugby and Cunamipreparations.

\section{LITERATURE}

[1] Janjić, V. R. (2000). Significance of ruderal aquatic flora and need for its destruction. Congress about weeds (VI), Koviljača spa, collection of work, pp. 40-51.

[2] Ješić, D., Medurić, D., Bauk, S., Ebel, F. (1981). The problem of weed on railways and possibility of destruction. In: Yugoslavian counseling about pesticides use, Colection of work, Opatija, pp. 535-539, Notebook 3.

[3] Kojić, M., Stanković, A., Čanak, M. (1972). Weeds - biology and destruction, Novi Sad: Faculty of agriculture - Institute for plant protection.

[4] Kojić, M., Šinžar, B. (1985). Weeds, Belgrade: Scientific book.

[5] Mitić, N.V., Savčić-Petrić, S.Lj. (2002). Pesticides in agricultures and forestry in Yugoslavia 2002 Belgrade: Society for plant protection of Serbia. 